\title{
El escultor José Rodríguez Díaz, «Sócrates» (h. 1746-1817)
}

\author{
M. a TeRESA CRUz YÁbAR
}

\begin{abstract}
RESUMEN
ABSTRACT

El escultor gallego José Rodríguez Díaz (San Pedro de Sesterey, Lugo

h. 1746-La Carraca. Cádiz 1817), formado en la Real Academia de Bellas Artes de San Fernando entre 1766 y 1785 , destacó como retratista de importantes personajes de la Corte. En este trabajo se dan a conocer su vida y obra, se identifican como suyos el busto del Conde de Floridablanca (Museo del Prado) y los de Manuel Ventura Figueroa y Miguel de Múzquiz (Real Academia de San Fernando), así como un par de relieves que existen en esta institución; se añaden a su catálogo obras desaparecidas como los modelos de Pelayo y Favila, dos leones y dos trofeos que decoraban la fachada del Monumento de Covadonga diseñado por Ventura Rodríguez, y un busto de Pedro Rodriguez de Campomanes.

The Galician sculptor José Rodriguez Díaz (San Pedro de Sesterey, Lugo h.1746-La Carraca, Cádiz 1817), who learnt in the spanish San Fernando Royal Academy of Art in Madrid between 1766 and 1785, stood out as portrait sculptor of outstanding members of the royal court. In this article about his life and artistic career, some pieces have been identified as his work: The bust of the Count of Floridablanca (Prado Museum), and those of Manuel Ventura Figueroa and Miguel de Múzquiz (Real Academia de San Fernando), as well as some reliefs owned by the same institution. Furthermore, six models belonging to Pelayo and Favila, to a couple of lions and to two throphies, which decorated the facade of the Monument of Covadonga - designed by Ventura Rodriguez- and a bust of Pedro Rodriguez de Campomanes, all missing, are included in the sculptors catalogue.
\end{abstract}


El escultor José Rodriguez Diaz nació en San Pedro de Sesterey, provincia de Lugo, hijo de Antonio Rodríguez y María Díaz ${ }^{1}$, hacia 1746. Aunque el Catálogo de Ázcue dice que había nacido en $1748^{2}$, las actas de la Real Academia refieren que tenía veinte años en $1766^{3}$ y veintidós en $1768^{4}$. Se matriculó en 1765 y comenzó a asistir a las clases de Juan Pascual de Mena ${ }^{5}$; en 1797 asegura, en cambio, que había aprendido el arte con Felipe de Castro ${ }^{6}$, lo que no parece exacto, aunque es muy posible que llegara a tener más adelante una buena relación con este profesor. Fue un alumno aplicado que participó asiduamente en las actividades académicas. Tomó parte en los concursos de 1766 ${ }^{\gamma}, 1769$ —donde obtuvo un primer premio de segunda clase con el relieve de pensado Fer-

\footnotetext{
Archivo de la Real Academia de Bellas Artes de San Fernando (RASF) 13-7/1, Certificación de Ignacio de Hermosilla a 6 de julio de 1768 .

L. Azcue Brea, La escultura en la Real Academia de Bellas Artes de San Fernando, Madrid 1994, 295, que hace de él la semblanza siguiente: "Estudió como alumno en la Real Academia Obtuvo el primer premio de segunda clase en 1769. Llegó a ser Académico de mérito en 1785 tras presentar un relieve del Martirio de San Esteban. Fue el director de las obras de escultura del Real Astillero de Cádiz". Otro autor que le dedica alguna atención es MARTin GONZÁLZ, J. J., Los escultores de la Real Academia de San Fernando, en IV Jornadas de Arte. El arte en tiempo de Carlos III, Madrid 1989, 261; da cuenta de que ganó dos premios, el primero de segunda clase en 1769 y el segundo de primera clase en 1781, que fue nombrado Académico de mérito el 6 de marzo de 1785 e identifica la Alegoria del nacimiento del Infante Carlos Eusebio que queda completa en la Academia de San Fernando (E-304) con la de este escultor.

El error sobre la fecha de nacimiento parece que procede de unas Papeletas de principio de siglo xx (RASF, 422-2/5) que dicen lo siguiente: “Rodriquez Díaz, D. José, nació en Lugo el año 1748 discípulo de esta Real Academia y Académico de Mérito de la misma en 6 de marzo de 1785. Murió en Cádiz en 21 de septiembre de 1817 \%. A su vez, el anónimo autor de la papeleta pudo haber hecho un cálculo sobre la edad de 33 años con que se decia que habia ganado una segunda medalla en 1781, según Distribución de los premios concedidos por el Rey nuestro señor a los discipulos de las tres Nobles Artes hecha por la Real Academia de San Fernando en la Junta Pública de 14 de julio de 1781 , Madrid 1781 , (Imprenta de la viuda de lbarra), 31 , sin tener en cuenta que el escultor la había reducido en dos años, quizá por haber concurrido al premio con
} dos escultores bastante más jóvenes.

3 RASF, 3/82, f. 376, junta general de 23-8-1766.

4 lbidem, f. 465v, junta ordinaria de 10-4-1768. En este acta se dice también que llevaba tres años de estudios; el dato de que se matriculó en 1765 consta también en la certificación citada en nota 1

5 V. nota 1. Ei mismo escultor afirma al pedir la certificación que estaba en la clase de Mena y lo confirma aquélla.

6 RASF, 173-1/5, Memorial de 24-3-1797: «...desde la edad de 14 años ha tenido el honor de estar bajo la protección de V.E., haviendo devido los primeros rudimentos y reglas al acreditado profesor don Felipe de Castro y otros a cuios lados y la continua asistencia a esta Academia adquirió los conocimientos necesarios a su profesión...". Es inexacto el dato de que había ingresado en la Academia a los 14 años, puesto que tenía 19. Lo más probable es que hubiera realizado algún aprendizaje antes de venir a Madrid.

7 RASF, $3 / 82, \uparrow .329$, junta general de 5.1-1766, elección de la prueba de pensado: copia del Mercurio grande de la Academia en bulto en yeso; Ibidem, 371, junta general de 23-7-1766 (prueba de repente: Baco joven de la Academia); Ibidem, f. 375, fallo de los premios. 
nando I arma caballero al $\mathrm{Cid}^{8}-, 1772^{9}, 1778^{10}$ y 1781 ; en este último obtuvo una segunda medalla en la primera clase con el relieve de pensado Alegoria del nacimiento del infante Carlos Eusebio ${ }^{11}$. Se conservan en la Academia el relieve de 1769 y dos fragmentos del que hizo en 1781. Consiguió también numerosas ayudas de costa por sus trabajos de dibujo del Natural y Escultura a lo largo de los dieciséis años que permaneció en las aulas y presentó voluntariamente varias obras de escultura ante la Junta de la Academia para demostrar sus progresos ${ }^{12}$. El 6 de marzo de 1785 fue designado académico de mérito tras haber presentado un relieve del Martirio de san Esteban ${ }^{13}$.

Su obra conservada es escasa y apenas se han hallado noticias de ella. En 1769 fue admitido en el grupo de escultores académicos que intervinieron en la reforma del retablo del Colegio Imperial tras la expulsión de los jesuitas, si bien con una participación de poca importancia que consistió en limpiar y hacer algunos atributos para los santos labradores de Manuel Pereira que se trajeron de la capilla de San Isidro en San Andrés ${ }^{14}$. Antes de su designación como académico de mérito debió hacer

8 RASF, 3/82, 1. 555-556, junta general de 27-6-1769. El asunto de pensado fue El Rey Don Fernando el Grande arma Cavallero al Cid Rui Diaz de Vivar, y el de repente. Sansón desquijarando al león.

9 RASF, 3/83, f. 130v.-131, junta general de 2-7-1772; concurrió con José Martinez Reina a las medallas de primera clase y quedaron las dos vacantes; el tema de pensado fue el Bautismo del Intante.

10 RASF, 3/84, f. 88v., junta general de 13-7-1778: el asunto de pensado fue Alfonso VI recibe el homenaje de los capitanes del Cid que le presentan treinta moros con sus caballos enjaezados, y el de repente fue Abraham y los tres ángeles; concurrió con Manuel Llorente, que ganó la primera medalla por catorce votos y Rodríguez Díaz no obtuvo ni un solo voto para la primera, ni tampoco para la segunda medalla, que quedó vacante. Ambos relieves (barro, tres cuartas de alto por vara y cuarta de ancho) quedaron en la Academia, y aparecen inventariados en lbidem, 617/3, Inventario 1804, †. 112v. con los números 163 (Rodriguez Diaz) y 164 (Manuel Llorente).

11 Distribución de los premios... 1781 cit., 18-19, 26 y 31; Cosme Velázquez ganó la primera medalla de la primera clase, mientras Martín Gutiérrez y José Rodriguez Diaz obtuvieron dos se. gundas medallas.

12 Los datos sobre ayudas de costa, que son muy numerosos, se incluyen en un trabajo más largo sobre este escultor que tenemos en preparación. Fueron tantos, que en la junta ordinaria de 5 12-1779 (RASF, 3/84, f. 140) se acordó que Rodriguez no tuviera ya ninguna opción a ayudas de trabajos mensuales, porque era conveniente que se estimulara también a otros discípulos.

13 RASF, 173-1/5. « Joseph Rodríguez Diaz, ... a los pies de Vuestra Excelencia con el devido respeto presenta un bajo reliebe que representa el Martirio de san Estevan que, junto con el grupo del mes pasado, a tin de que si V.E. tuviese a bien el que se le reciva por uno de los Academicos de Mérito, se digne a executarlo, por cuya gracia quedará el suplicante agradecido y obligado a intentar mayores progresos y adelantamientos. Favor que espera de la bondad de V.E. el suplicante. Madrid 4 de marzo y de 1785 ".

14 Archivo Histórico Nacional, Clero, Jesuitas, leg. 653, (Cuenta de gastos de pintores y escultores que trabajaron en el retablo del antiguo Colegio Imperial, convertido en iglesia de San Isidro. Año 1769). Participaron en la reforma del retablo el pintor Mengs y los escultores Mena, Álva- 
pequeñas obras para ayudar a su sustento, como una cabeza y manos de un Santo Tomás encargado por un particular que presentó a la Academia en $1778^{15}$.

Por una noticia que dio Manuel Martín Rodríguez sabemos de una participación del escultor en la obra de las fuentes del Prado por la que Ventura Rodríguez había mandado que se le pagase el 26 de noviembre de 1782; y que años más tarde, el 31 de diciembre de 1785, hacía una puja junto con Pablo de la Cerda (o Pablo Çerdá) para realizar lo que faltaba en la fuente de Neptuno, que era la carroza en forma de concha, el agua y los delfines, poniendo la obra en 60.000 reales. Aunque no les fue adjudicada en un principio, el 26 de marzo siguiente, después de haberse puesto de acuerdo con José Guerra, el Ayuntamiento de Madrid contrató con los tres la obra en 47.000 reales ${ }^{16}$. Algo más tarde realizó un proyecto para hacer un segundo cuerpo de madera en el trascoro de la catedral de Ávila. Presentado a la Comisión de Arquitectura de la Academia, ésta lo reprobó en agosto de $1786^{17}$.

Entre 1785 y 1791 transcurrió el periodo más fructífero de la vida del escultor, al haber disfrutado de la protección de Pedro Rodríguez de Campomanes, presidente del Consejo de Castilla. Quizá fue Felipe de Castro quien dio a conocer al ilustrado asturiano a este escultor joven, casi paisano suyo, que demostraba habilidades como retratista. Campomanes y Castro tenían una buena amistad, reforzada por su común admiración por el padre Sarmiento. En cualquier caso y cualquiera que sea el camino por el que conoció al Gobernador del Consejo, Rodríguez Díaz recibió el encargo de hacer su retrato y el de varios personajes de la política afines al asturiano, vivos o fallecidos. Que sepamos, además de don Pedro, retrató a Ventura Figueroa, predecesor de Campomanes en la presidencia del Consejo, a Miguel de Múzquiz, ministro de Hacienda, y al primer ministro Floridablanca. No es probable que se le hicieran los encargos antes de

rez, Gutiérrez, Juan Martínez Reina, Manuel Bergaz, Arali, Rudiez e Isidro Carnicero además de Rodriguez Díaz.

RASF, $3 / 84, f$. $71 v$, junta ordinaria de 18-1-1778.

16 Archivo de la Villa de Madrid, A.S.A. 1-117-5. Cfr. Diaz y DiAZ, M.S., Noticias sobre algunas fuentes monumentales del Madrid del siglo XVIII, "Villa de Madrid" 54, (1977), 57 y BLANCo Mozo, J.L., La cultura de Ventura Rodriguez. La biblioteca de su sobrino Manuel Martín Rodriguez, "Anales del Departamento de Historia y Teoria del Arte (U.A.M.)", VII-VIII (1995-1996), 181-231. Este último autor cita el protocolo donde se halla el contrato (A.H.P.M., 20.060, f. 520-525).

17 Documentos para la Historia de Ávila (Museo Provincial de Ávila), Ávila 1985 (RASF, Actas de la comisión de Arquitectura 1786-1805, 1/139, f. 36; Informes Comisión de Arquitectura 17581815, 1-28/5); Garcia Melero, J.E., Las catedrales góticas en la España de la llustración, Madrid 2001, 154-155. 
que hubiera obtenido la graduación como académico de mérito, por lo que pensamos que han de datarse a partir de 1785. El único que al parecer se conserva en su versión definitiva es el de Floridablanca del museo del Prado, en tanto que la Academia de San Fernando guarda los modelos en yeso de Múzquiz y Figueroa y tenía también el de Campomanes, actualmente perdido ${ }^{18}$ y que muy posiblemente sería el modelo para el busto en mármol que tenía el duque de Valencia en $1902^{19}$.

Por igual vía debió llegarle en 1789 el encargo de la parte más importante de la escultura para el nuevo Monumento de Covadonga. Recientemente se ha confirmado que fue Campomanes el defensor más ardiente del proyecto de Ventura Rodríguez para el edificio que se habia de levantar al lado de la gruta de la aparición de la Virgen. Monumento que sus historiadores, y especialmente Reese ${ }^{20}$, coinciden en afirmar de carácter laico, puesto que se destinaba a guardar los restos de Pelayo, pero no la imagen de la Virgen, ya que los canónigos de la Colegiata se oponían a su traslado desde la cueva. El proyecto avanzaba lenta y costosamente ya muerto su creador cuando el Consejo encargó al sobrino de Ventura, el arquitecto Manuel Martín Rodríguez, que supervisara las obras, a cuyos efectos viajó a la Colegiata y emitió un informe el 25 de septiembre de 1789. A su vuelta debió tratar con Rodríguez Díaz para que hiciera la parte más notable de la escultura: las dos grandes efigies de Pelayo y Favila, que iban colocadas en unos nichos en el pórtico tetrástilo que se abría en la segunda planta del edificio, los dos leones que adornaban el arranque de la escalinata en la base y los dos trofeos de cuatro caras para el final de la balaustrada, que son visibles en la fotografía publicada por Chueca de uno de los dibujos del proyecto de Ventura Rodríguez, hoy en paradero ignorado (Fig. 1). Encomendó otra parte de la escultura al también académico de mérito José Piquer, que haría el escudo sostenido por la Fama y la Gloria que adornaba la puerta de acceso en el piso inferior y el medallón de la Virgen sobre el hueco de entrada al pórtico, así como los dos ángeles que flanqueaban el Tabernáculo en el interior. Un tercer escultor

18 RASF, 617/3, Inventario 1804: “...Bustos, cabezas y mascarillas de yeso: ...113. Busto de Campomanes de Joseph Rodríguez Díaz".

19 Un busto en mármol de Campomanes figuró con el número 1673 a nombre de José Rodriguez y se fechó en 1787 en el catálogo de la Exposición Nacional de Retratos celebrada en Madrid en 1902 (Ministerio de Instrucción Pública y Bellas Artes, 232). En la actualidad se ignora el paradero tanto del modelo como de la probable versión definitiva.

20 Sobre la intervención de Campomanes, v. MADRID Ál VAREZ, V. de la, El proyecto de Ventura Rodriguez para el santuario de Covadonga, "La nueva España" 2-2-2002; REESE, T.F., V. Rodriguez, Jovellanos y Covadonga: proto-romanticismo en la España del siglo xvill, "Archivo Espanol de Arte" 197 (1977), 31-58. 


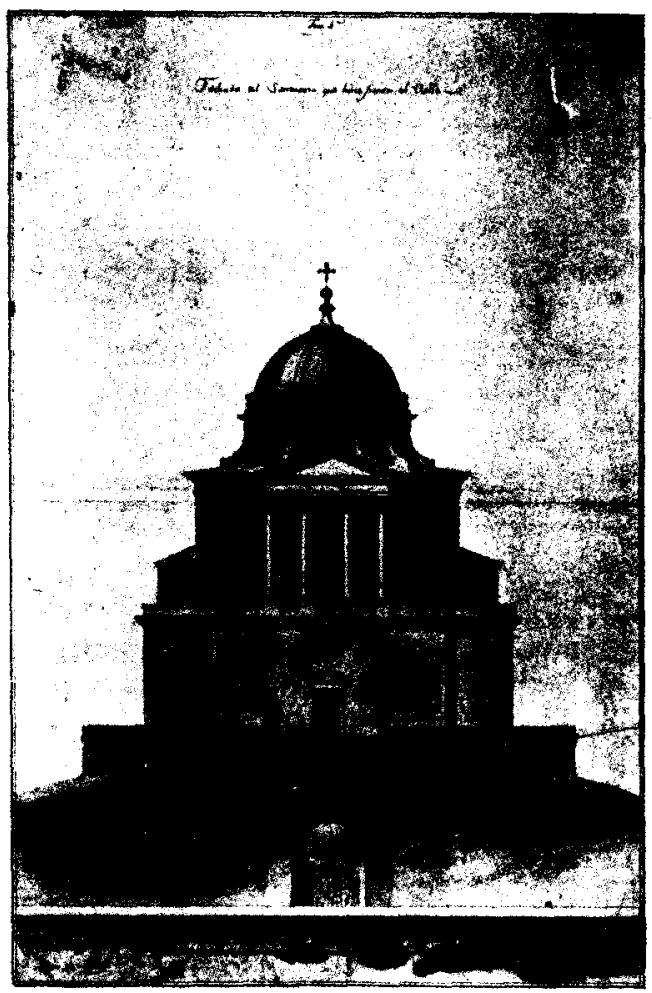

Fig. 1. Ventura Rodriguez. Proyecto de la fachada del monumento de Covadonga (dibujo publicado por Fernando Chueca).

académico, Francisco Meana, intervendría también con un encargo de menor importancia que los anteriores ${ }^{21}$.

El 21 de septiembre de 1790, Rodríguez Díaz presentó al Consejo un memorial y unos modelos de pequeño tamaño, seis en total, evaluando las futuras esculturas en una cantidad cercana al medio millón de reales $^{22}$. Los elevados gastos que estaba ocasionando la construcción del

21 Canella Secades, F., De Covadonga (Contribución al XII Centenario): Santuario de Auseva, el Conde de Campomanes y el arquitecto Rodriguez, Madrid 1918, y CHUECA GoITIA, F., Dibujos de Ventura Rodriguez para el Santuario de Nuestra Señora de Covadonga, "Archivo Español de Arte" 56 (1943), afirmaron que José Piquer hizo la escultura de Covadonga sin mencionar siquiera a Rodríguez Díaz.

22. RASF, 27-1/2; el memorial al Consejo de Castilla de 21-9-1790 dando cuenta del precio en que estaba dispuesto a ejecutar la obra definitiva, y el de 22 de mayo de 1791 en que el escultor 
Monumento y, sobre todo, la dimisión de Rodríguez Campomanes el 19 de abril de 1791 y la caída de Floridablanca en febrero de 1792 determinaron la suspensión del proyecto a fines de este último año. Se decidió despedir a los escultores pagándoles sus modelos. Martín Rodríguez tasó los de Rodríguez Díaz en 7.000 reales, con lo que dio lugar a una larga polémica con el escultor -que le acusaba de haberle prometido 20.000 reales por ellos - y que terminó con su retasa por el director general de la Academia, Manuel Álvarez, y el teniente director de Escuitura Isidro Carnicero, que los evaluaron en 14.200 reales $^{23}$. Los modelos quedaron depositados en la Academia y allí se conservaban en sus cajones en $1804^{24}$, aunque nada se sabe ahora de ellos.

En 1793, después de servir en la Academia como profesor ayudante de las clases de Principios desde 1786, Rodríguez Díaz aspiró a la plaza de profesor de Anatomía sin obtenerla. En sus años juveniles se habia distinguido especialmente en esta asignatura, lo que reconocieron los profesores, como él mismo afirma, "premiándole muchas veces y honrándole con el nombre de Sócrates" 25. En 1797 optó a la sucesión de Alfonso Bergaz como teniente director de Escultura, pero consiguió sólo un tercer lugar en la terna ${ }^{26}$.

El trabajo no abundaba, tanto que se vio precisado a buscar acomodo en un puesto oficial. A fines de aquel año logró ser nombrado maestro mayor de escultura en el Arsenal de la Carraca, en Cádiz, con sueldo dia-

se queja del precio en que se han tasado los modelos se vieron en la junta ordinaria de la Academia de 5 de junio de 1791

23 RASF, 3/124, f. 191, junta ordinaria del 3-6-1792.

24 RASF, 617/3, Inventario 1804, f. 161: “Obras depositadas en la Academia... Seis caxoncitos, dos señalados Rey Fabila, infante don Pelayo, dos con trofeos y otros dos con leones echos para Cobadonga por don Joseph Rodríguez Díaz...". También se conservaba el motivo de la decoración de la pueria de ingreso al monumento hecho por Piquer, que el Gobernador del Conse. jo consiguió que el juez ante el que se tramitaba la testamentaría del escultor después de su muerte en 1794 lo hiciera depositar en la Academia (La petición en RASF, 33-1/2, junta ordinaria de 5-3-1796; el modelo en 617/3, Inventario 1804, «161v: Dos modelos en yeso de dos escudos de Castilla y León con dos genios que le sostienen, el uno tiene la corona separada, dos figuritas sueltas y parte del molde, por don Joseph Piquer, depositadas en la Academia por el señor Teniente de la Villa, don Juan Antonio de Santa María").

25 RASF, $21-1 / 1$, Petición para que la Academia le encargue la comisión de enseñar las proporciones del cuerpo humano, de 5-10-1793. El memorial se inicia: «Excelentísimo señor: don Joseph Rodríguez Diaz, conocido por Sócrates...". El lucense habia presentado ante la Junta un esqueleto redondo hecho en barro (RASF, 3/82, ‘. 482v., junta ordinaria de 5-6-1768), tal como deseaban Mengs y Castro que practicaran los alumnos de Agustín Navarro, el profesor de Anatomía (BÉdat, C., La Real Academia de Bellas Artes de San Fernando, 1744-1808, Madrid 1989, 150-151).

26 RASF, 173-1/5, memorial de 24-3-1797. Los pormenores de la elección en Fábrica Nacional de Moneda y Timbre (F.N.M.T.), Diario del grabador Pedro González de Sepúlveda, libro IV, 1797-1802, fol. 18, donde confirma que entre sus compañeros era conocido como "Sócrates". 
rio de 30 reales $^{27}$, y el 1 de febrero de 1798 escribía a la Academia pidiendo una ayuda para sus gastos de traslado, pues su economía estaba maltrecha por no haber tenido encargos de obras en siete años ${ }^{28}$, esto es, desde que su protector salió del gobierno. Llegó a La Carraca el 11 de abril siguiente ${ }^{29}$. El 5 de octubre de 1803 se hallaba de vuelta en Madrid, porque a principios de ese año había sido despedido a causa de «la variedad de pareceres que tiene el Ministerio de Marina» ${ }^{30}$. Pleiteó para recuperar su plaza hasta que consiguió su objetivo, comunicando a la Academia el 4 de mayo de 1805 que regresaba al Arsenal. Sócrates moriria en Cádiz el 21 de septiembre de $1817^{31}$. Nada se conoce de lo que hizo allí, ni siquiera de lo que debió ser su cometido oficial, los mascarones de proa y otros adornos escultóricos para los buques de guerra. Cabe suponer que ejerció de retratista por sus buenas dotes en este género, en el que no brillaban en cambio Cosme Velázquez y José Fernández Guerrero, sus antiguos compañeros de la Academia, que regentaban la de Bellas Artes de Cádiz, pero no hemos localizado resto alguno de su producción en estos últimos años.

Catalogamos a continuación las obras que conocemos del escultor.

\section{FERNANDO I ARMA CABALLERO AL CID. 1769 (Fig. 2).}

Relieve en barro cocido

$35 \times 72 \mathrm{~cm}$.

Le falta la parte superior, desde la cabeza de los personajes hacia arriba. Abajo, a tinta, «91».

Real Academia de Bellas Artes de San Fernando, E-307.

El Catálogo de Ázcue incluye con la referencia citada este relieve, correspondiente a la prueba de pensado de 1769, y sigue la tradición que lo atribuye a José Martínez Reina, uno de los opositores del concurso ${ }^{32}$. Según dicho Catálogo, el Inventario de 1804 de la Academia recoge en la partida 91 - número que luce la pieza-, un relieve en barro con el siguiente texto: "91...El Rey don Fernando el Grande armando caballero al

27 RASF, 173-1/5.

28 Ibidem.

29 Ibidem. «Señor don Ysidoro Bosarte... He llegado a esta tierra el día 11 del pasado y me ba vien en ella de salud, que lo más, está tan mal como allá quedare...en la Ysla de León y abril 20 de 98. Josef Rodriguez Díaz". Bosarte era secretario de la Academia y debió ser uno de los que abogaron a favor de que se le concediera el puesto de La Carraca

Ibidem.

31 RASF, 422-2/5, Papeletas de principios del siglo $x x$.

32 RASF 3/82, 千. 555, junta general de 27-6-1769: «Formóse la Junta a las ocho y media de la mañana y leido el acuerdo de la antecedente, presentaron los profesores de Escultura varios asumptos para las pruebas de repente de cada una de las clases y aprobados un considerable 


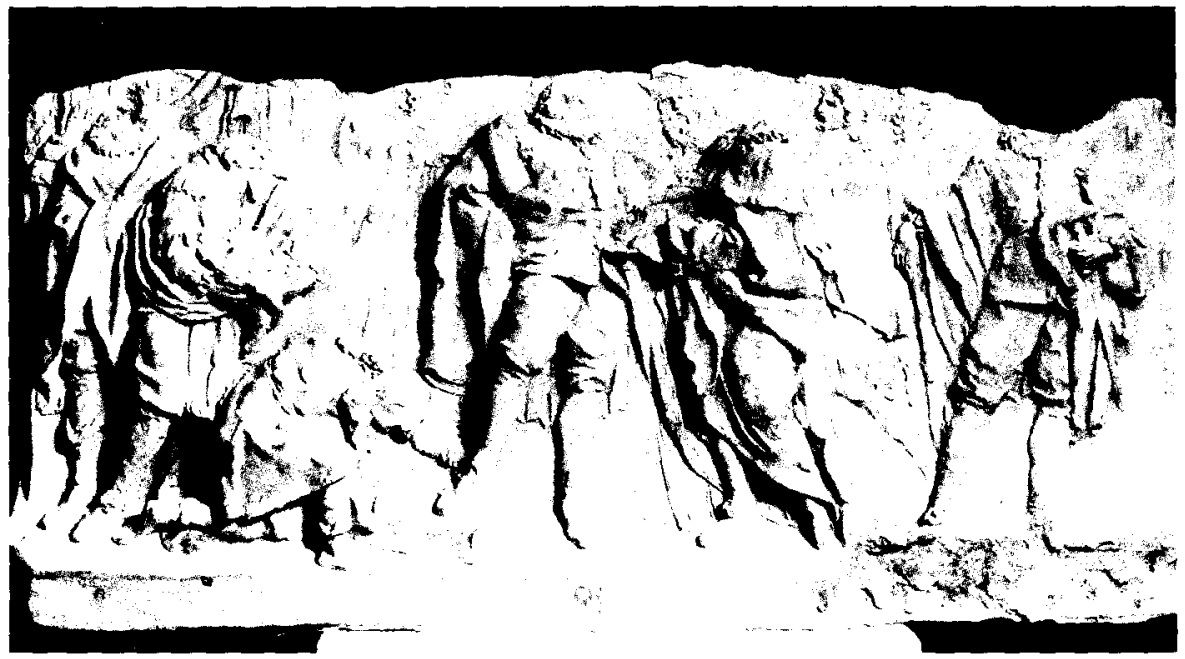

Fig. 2. José Rodriguez Diaz. Fernando I arma caballero al Cid. 1769 (Real Academia de San Fernando).

Cid Rui Díaz, que obtuvo el premio primero de segunda clase en el año 1769...». Y añadido: «D. José Martínez Reina» ${ }^{33}$. Sin embargo, el ejemplar del Inventario de 1804 que hemos consultado dice bien claramente que el número 91 corresponde a la obra ejecutada por José Rodríguez Díaz ${ }^{34}$. El

número se eligieror los siguientes:...Para la segunda clase, practicando lo mismo, salió por suerte Quando Sampson desquixaró al león.

Separóse la junta (aprobados estos asuntos), retiráronse los profesores y los señores marqueses de Santa Cruz, presidente, don Joseph de Hermosilla, don Francisco Xavier Carrión, el Director general y yo. pasamos a distribuir los referidos asumptos en las Salas de principios a los opositores de todas clases, dándoles a cada uno su tablero con barro para executar la prueba y ios que se presentaron fueron los siguientes: en la primera clase, don Alphonso Vergaz-A; don Joseph Arias-B; Pablo Serdá-C. En la segunda clase, don Joseph Martinez Reyna-D; Joseph Rodríguez Diaz-E. En la tercera clase, Ygnacio Dabouzada-F; Miguel Moço-G; Martín Gutiérrez-H; Pedro del Mazo-Y.

[f. 556] A las onze y media se bolbió a formar la junta, se recogieron las pruebas en la forma acostumbrada y de los vocales concurrentes se hallaron hábiles para votar los premios a los señores don Antonio González, don Phelipe de Castro, don Juan Pasqual de Mena, don Roberto Michel. don Joseph Grichi, don Manuel Álbarez, don Francisco Gutierrez, don Pedro Michel, don Santiago Labán y don Ysidro Carnicero....En la segunda clase, de los diez vocales, seis estubieron a favor de la letra $E$, quatro a favor de la $D$, vajo cuio sisthema se adjudicó el primer premio de esta clase a don Joseph Rodríguez Díaz, a quien correspondía la letra $E$, y el segundo a don Joseph Martinez Reyna, bajo la letra D, que fueron los únicos opositores de esta clase..."

33 AzCUE BREA, L., op. cit, 247-248.

34 RASF, 617/3: "Baxos relieves... [f. 102]: «91. El rey Don Fernando el Grande armando Caballero al Cid Rui Diaz de Vivar por don Joseph Rodríguez Diaz, que obtuvo el premio primero 
escultor Martínez Reina no ganó el primer premio, sino el segundo, por lo que, si era su obra la que llevaba el número 91, el asiento debia haber dicho "premio segundo" y no "premio primero"; en nuestra opinión, la confusión no está en el número ni en la categoría del premio, sino en el nombre del escultor, añadido en un momento indeterminado en alguna copia del Inventario de 1804 y no en otra. Confirma esta hipótesis el hecho de que el Inventario de 1824 diga también que el relieve correspondiente a Rodríguez Díaz está señalado con el número 91 y el de Martínez Reina con el 120. El ejemplar de la prueba de este último, al parecer, no se conserva. La atribución errónea al escultor murciano de la pieza E-307 se ha venido repitiendo desde entonces y así la recogen Serrano Fatigati, Baquero Almansa, Ázcue y Martín González ${ }^{35}$.

El escultor utiliza figuras de gran tamaño que llenan totalmente la escena en primer término, sin dejar entre ellas espacio que permita adivinar el lugar en que se sitúa la acción; es posible que contribuya a este efecto de ausencia espacial la pérdida de la parte superior del relieve, en que podía existir alguna mayor referencia de paisaje o escenario. Destaca la técnica abocetada y la escasa gradación del relieve entre las figuras del primer y segundo término, que pasan bruscamente del altorrelieve al schiacciato. Produce la impresión de ser una obra preparatoria en que el escultor tratara de reflejar su invención de un modo somero y rápido. El Cid y el rey se sitúan aproximadamente en el centro y no están destacados por tamaño o por altura de relieve respecto de los otros personajes. Visten a la usanza de la época de Felipe II, lo que nos lleva a pensar que Rodríguez Díaz no había asimilado las enseñanzas de Felipe de Castro respecto de la necesidad de documentarse debidamente para evitar anacronismos, sino que asistía aún a las clases de Juan Pascual de Mena. En nuestra opinión, es una obra relativamente original dentro de las pautas del relieve académico de pensado, en que los alumnos trataban de demostrar su virtuosismo en la perspectiva, en el equilibrio compositivo y en la perfección de un acabado pulido, rasgos ausentes en esta obra, que en cambio destaca por una cierta espontaneidad y vigor de que carecen otras semejantes.

de segunda clase en el año de 1769, alto dos tercias, ancho tres quartas y media...; [f. 107] 120. El asunto explicado en el n. 91 por don Joseph Martínez Reina, que obtuvo el segundo premio de segunda clase en 1769 ".

35 Seraano fatigati. E., Escultura madrileña desde mediados del siglo xvi hasta nuestros dias", "Boletín de la Sociedad Española de Excursiones" XVIII (1910), 247; BAQUERO ALmANSA, A., Profesores de Bellas Artes murcianos. Con una introducción histórica, Murcia 1913, 278., AZCUE BREA, L., Inventario de las colecciones de escultura de la RASF, “Academia" 62 (1986), 294; Martín González, J. J., op. cit, 264. 


\section{ALEGORÍA DEL NACIMIENTO DEL INFANTE CARLOS EUSEBIO. 1781 (Figs. 3 y 4 ).}

Relieve en barro cocido.

Tan sólo se conservan dos fragmentos correspondientes a los ángulos superior e inferior derecho.

$40 \times 55 \mathrm{~cm}$. y 43 x $38 \mathrm{~cm}$.

Real Academia de Bellas Artes de San Fernando, E-501.

El fragmento de relieve más grande, correspondiente al ángulo superior derecho, muestra un joven alado y un niño descabezado volando entre nubes y enmarcados por un cortinaje con flecos, cordones y borlas, y parte de un fuste estriado de columna a la derecha. El más pequeño, el del ángulo inferior derecho, permite ver el resto de un almohadón y otro trozo del fuste. El Catálogo de Ázcue lo recoge como de autor anónimo, si bien hace referencia en el texto a su posible identificación con alguno de los relieves presentados al concurso de primera clase de $1781^{36}$. Pensamos que los fragmentos son partes del relieve presentado por José Rodríguez Díaz a dicho concurso.

El asunto de pensado de primera clase se definió ese año de igual modo para la pintura y la escultura: "Un quadro alegórico, alusivo al nacimiento del Infante heredero del Reyno, en esta forma. En un país frondoso de árboles, entre los quales podrá divisarse, si se quiere, parte del Palacio del Pardo, será la principal acción recibir España entre ráfagas de luz al Infante Heredero con una mano y con la otra podrá alzar la cubierta de regia cuna, donde va a ponerle, figurándose ésta como basa o escalón de un trono, concurriendo la pintura en acción de toldar con un tapiz, en el cual podrá figurarse alguna de las victorias de los Españoles: la Escultura, que indique colocadas sobre un pedestal tres gracias, con alusión a las tres serenísimas infantas hermanas del recién nacido; la Arquitectura, puesto un brazo sobre vestigios de las columnas de Hércules y señalando la inscripción PLUS ULTRA, manifestará deberse esperar nuevas felicidades del Infante; se representará el río Manzanares reclinado sobre su taza, asistido de Ninfas, que tañerán cítaras o algunos otros instrumentos; un mancebo con alas y llama sobre la cabeza, como que baxa del cielo para servir de ayo al Infante y algunos geniecitos en el ayre, que le ofrezcan atributo de héroe " ${ }^{37}$.

Los opositores a la primera clase de escultura fueron tres, Cosme Velázquez, Martín Gutiérrez y José Rodríguez Díaz; el primero consiguió seis votos y obtuvo la primera medalla, mientras los otros dos tuvieron cuatro

36 AZCUE, op. cit., 374.

37 Distribución de los premios... 1781 cit., 18-19. 

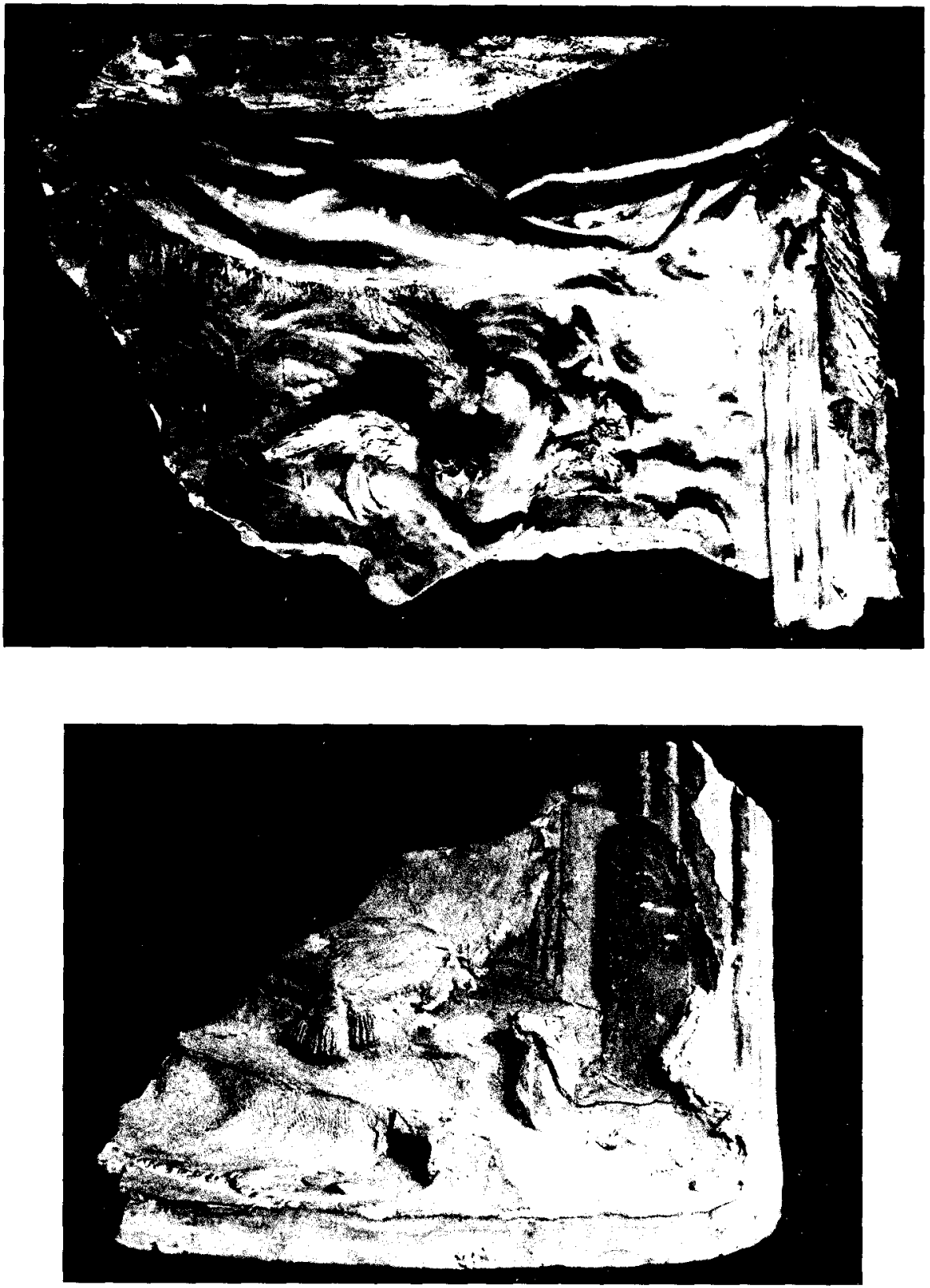

Figs. 3 y 4. José Rodriguez Díaz. Fragmentos de los ángulos superior e inferior derechos del relieve Alegoría del nacimiento del infante Carlos Eusebio. 1781

(Real Academia de San Fernando). 
votos cada uno y la Academia duplicó el segundo premio, que correspondió en primer lugar a Martín Gutiérrez por tener menos años de estudios y en segundo lugar a Rodríguez Diaz ${ }^{38}$.

Los inventarios más antiguos de la Academia reflejan la existencia de los tres relieves, marcados con el 141 el de Rodríguez Díaz, con el 137 el de Martín Gutiérrez y con el 146 el de Cosme Velázquez ${ }^{39}$.

Existe en la Academia un relieve muy bien conservado (E-304) que responde enteramente a esta escena (Fig. 5). El Catálogo de Ázcue admite como su posible autor a los tres escultores participantes en el concurso para la primera clase de escultura de 1781, Cosme Velázquez, Martín Gutiérrez y José Rodríguez Díaz, sin decidirse por ninguno de ellos. Ni éste ni los fragmentos de que tratamos parece que conserven ninguna indicación numérica. A nuestro entender el relieve completo tiene una factura excesivamente meditada, una gradación llena de matices y unas figuras demasiado pequeñas para que se trate de una obra del lucense; se atiene con rigor a todas las indicaciones con que se enunció el asunto, es muy equilibrado según el gusto académico y está trabajado cuidadosamente en altura, profundidad y detalles. En el relieve fragmentado, la escena parece más abocetada y los pormenores apenas están insinuados. La maestría de Cosme Velázquez en la composición y el acabado nos llevan a atribuir con preferencia al riojano el relieve completo, que sería el ganador del concurso, y a Rodríguez Díaz los fragmentos, por su factura rápida y algo descuidada y el tamaño relativamente grande de la única figura que ha quedado completa.

3. RETRATO DE MANUEL VENTURA FIGUEROA. 1785/1791 (Fig. 6).

Busto en escayola.

$53 \times 34 \times 20 \mathrm{~cm}$.

Buen estado de conservación

Sobre el plinto, a tinta, "118» y una etiqueta encima con «62»; en la basa una etiqueta con " $2 / 140$ ".

Real Academia de Bellas Artes de San Fernando, E-399.

38. Ibidem, 31.

39 RASF, 617/3, Inventario 1804, f. 109-110: «137. Alegoría al Nacimiento del Ynfante don Carlos Eusebio, Heredero del Reino, por Dn. Martín Gutiérrez, que obtuvo el segundo premio de primera clase en 1781, ancho vara y media quarta, alto dos tercias, faltan dos cabezas... 141. Alegoría al Nacimiento del Ynfante don Carlos Eusevio, heredero del Reyno, por don Joseph Rodriguez Díaz, que obtuvo premio segundo extraordinario de primera clase en 1781; alto dos tercias, ancho vara y media quarta... 146. Alegoria del Nacimiento del Ynfante heredero del Reyno en barro, primer premio de primera clase del año 1781, por don Cosme Velázquez, alto tres quartas y media, ancho vara y tercia". En el Inventario de 1824 tienen los números 2,4 y 26 y en los asientos se hace referencia a la numeración de 1804, que conservaban los relieves. 


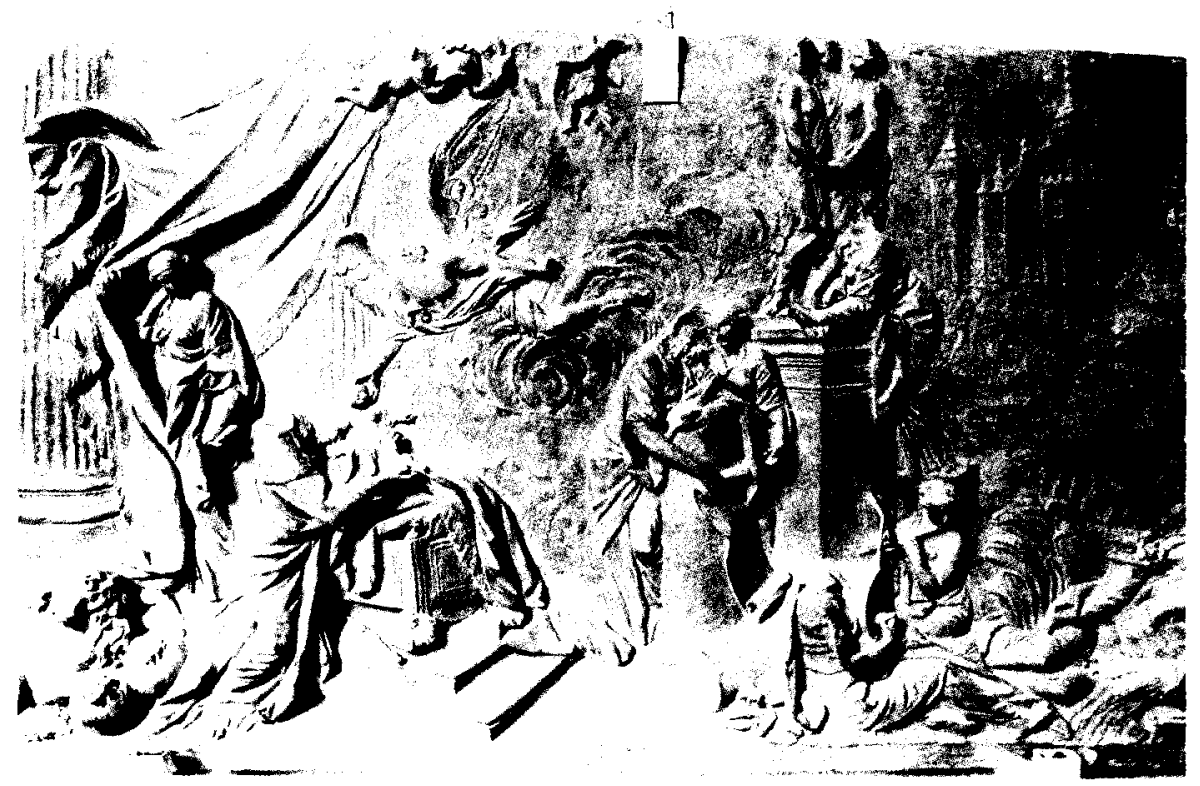

Fig. 5. Cosme Velázquez. Alegoria del nacimiento del infante Carios Eusebio. 1781 (Real Academia de San Fernando).

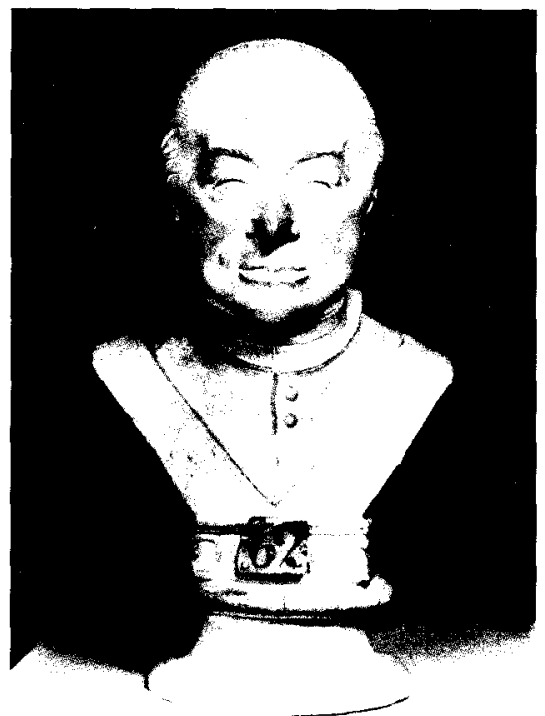

Fig. 6. José Rodriguez Diaz. Retrato de Manuel Ventura Figueroa. 1785/1791 (Real Academia de San Fernando). 
Cabeza y busto cortado triangularmente de eclesiástico vestido a la moda de fines del siglo XVIII. Se alza sobre un plinto de base cuadrada y basa de tipo ático.

Aparece identificado en los inventarios antiguos de la Real Academia como Manuel Ventura Figueroa, presidente del Consejo de Castilla, donde también se designa como su autor a Rodríguez Díaz. El Catálogo de Ázcue coloca interrogantes tanto al autor como al retratado pero, a nuestro juicio, ni lo uno ni lo otro ofrece duda atendiendo a los números $118 \mathrm{y}$ 62 que figuran en su plinto y son los mismos con que se identifica en el inventario de 1804 y catálogo de 1829 respectivamente como Ventura Figueroa y original de Rodríguez Diaz ${ }^{40}$.

Manuel Ventura Figueroa había estado unido por fuertes vínculos de amistad al padre Rávago, jesuita y confesor de Fernando VI, y a la Compañía, pero, en el momento en que se gestaba su expulsión, volvió la espalda a sus protectores por ambición personal. Sucedió al conde de Aranda en 1773 como presidente del Consejo de Castilla y Pedro Rodríguez de Campomanes fue su fiscal y sucesor tras su muerte en 1783. Llegó a ser patriarca de las Indias y limosnero mayor del Rey, arzobispo de Laodicea y comisario general de la Cruzada. Rodríguez Casado le tacha de "clérigo intrigante y venal» ${ }^{41}$.

Rodríguez Díaz captó de forma espléndida esta condición espiritual del Gobernador del Consejo, que Ázcue define más suavemente como "sagaz". Está dentro de lo posible que el busto fuera el modelo del que se colocó en el sepulcro del eclesiástico en la iglesia de San Martín de Madrid. Era costumbre entre los académicos presentar ante sus compañeros las obras importantes que hacian para sitios públicos y, en caso de escultores, depositar en la Academia los modelos que habian servido para ejecutarlas. Ponz señala en la tercera edición del tomo V de su Viage lo siguiente: “Después de la última edición de este libro se colocó en el ingreso de esta capilla (al lado del Evangelio) otra memoria sepulcral, que consiste en un busto y dos figuras alegóricas de niños, con este letrero en el pedestal: "(alfa y omega flanqueando un crismón) Aquí existen las cenizas del llustrísimo y Excelentísimo Señor don Manuel Ventura Figueroa, Arzo-

40 AzCUE, op. cit, 295. RASF, 617/3, Inventario 1804, f. 121: “118. Busto de Figueroa, gobernador del Consejo de Castilla, por Don Joseph Rodríguez Díaz». Catálogo 1824, f.70 (el mismo texto); Catálogo 1829, f. 80 «...62 El Excelentísimo Señor Don Manuel Ventura de Figueroa, por Don José Rodriguez Díaz".

${ }^{41}$ Rodriguez Casado, V., La politica y los politicos en el reinado de Carlos III, Madrid 1962 , 226-227 
bispo de Laodicea, Patriarca de las Indias, Pro Capellán, y Limosnero mayor de SM, Comisario general de la Santa Cruzada, Gran Cruz y Canciller de la Distinguida Orden de Carlos III, Gobernador del Consejo. Nació en Santiago a 23 de diciembre de 1708. Falleció a 3 de abril de 1783, en cuyo día se debe decir una misa rezada en esta iglesia por su alma. RIP". La escultura de este Sepulcro la hizo el profesor D. Joseph Díaz" 42.

Fechas límites para este retrato son, por tanto, las de 1782 y 1793 , en que aparecen respectivamente la segunda y tercera edición del tomo $\mathrm{V}$ de Ponz relativo a Madrid, aunque lo más probable es que fuera encargado después de la muerte de Ventura Figueroa con la finalidad de decorar su sepulcro; las consideraciones que hicimos más arriba respecto a la cualidad de académico que tendría Rodríguez Díaz al recibir el encargo -el texto de Ponz le califica de "profesor»- hace preferible reducir este periodo a los años posteriores a 1785; en cuanto al límite superior, debemos fijarlo en 1791, año de la dimisión de Rodríguez de Campomanes. Seguramente se hizo más cerca de 1785 , teniendo en cuenta la fecha de fallecimiento del retratado.

Fernando Selma grabó una estampa con la efigie de Ventura Figueroa según el busto en mármol que hizo Rodríguez Díaz ${ }^{43}$.

4. RETRATO DE MIGUEL DE MÚZQUIZ, CONDE DE GAUSA. 1785/1791 (Fig. 7).

Busto en escayola.

$52 \times 29 \times 25 \mathrm{~cm}$.

Buen estado de conservación.

Sobre el plinto, a tinta, "114"; en la basa una etiqueta con «2/119".

Real Academia de Bellas Artes de San Fernando, E-469.

Cabeza y busto cortado en forma aovada de persona avanzada en años, que adorna su cabeza con peluca rizada y corta, y viste casaca y corbatín de abundante plegado a la moda de fines del siglo XVIII. Plinto y basa como el anterior.

Es efigie del ministro de Hacienda de Carlos III, Miguel de Múzquiz, fallecido en 1785. El Catálogo de Ázcue lo data entre 1780 y 1785, quizá teniendo en cuenta la fecha de la muerte del político. En nuestra opinión,

Ponz, A., Viage de España, V, 3. ${ }^{3}$ ed., Madrid, 1793, 213-214.

43 PAEz Rios, E., lconogratia Hispana. Catálogo de los retratos de personajes españoles en la Biblioteca Nacional, Madrid 1966, n." 3207; mide $110 \times 164 \mathrm{~mm}$. La leyenda dice: "El Excmo. e Iltmo. Sr. D. Manuel Ventura Figueroa, Patriarcha de las Indias, Governador del Consejo", y al pie, "Josef Rodriguez lo esculpió en mármol» y "Fernando Selma lo grabó". Las fechas de nacimiento y muerte del escultor en este catálogo (1839-1909) son incorrectas. 


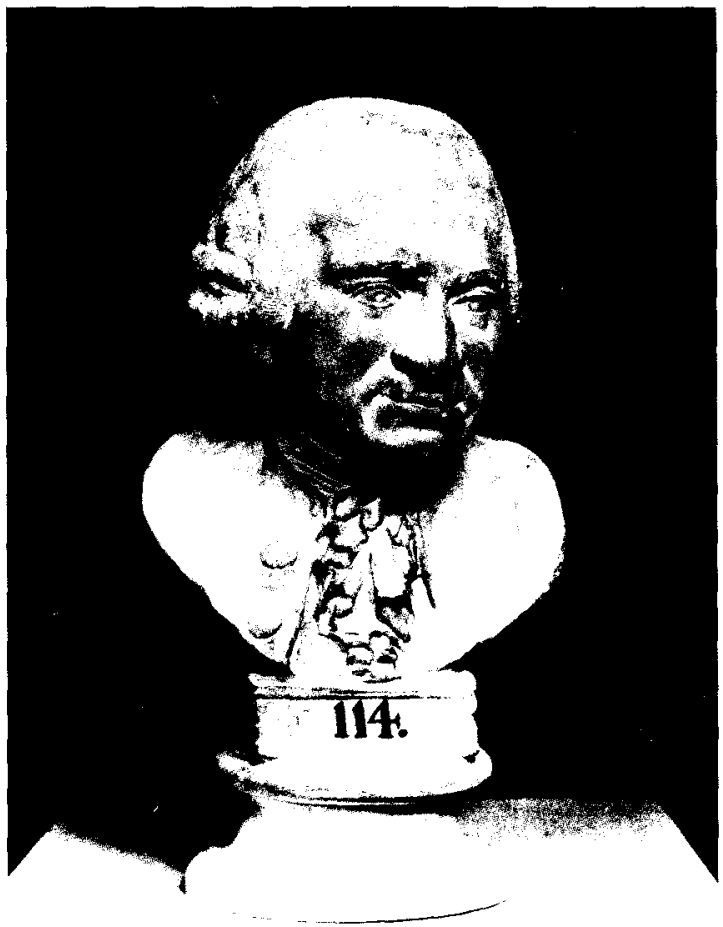

Fig. 7. José Rodriguez Diaz. Retrato de Miguel de Múzquiz, conde de Gausa. 1785/1791 (Real Academia de San Fernando).

es posiblemente un retrato póstumo realizado a partir de alguna pintura - de otra escultura, ya que cuando murió el ministro, José Rodríguez Díaz acababa de conseguir el grado de académico de mérito y parece un poco prematuro que obtuviera antes el honor de retratar a tan importante personaje.

El busto aparece en los inventarios más antiguos de la Academia perfectamente identificado en cuanto al retratado y a su autor, y conserva aún en su pedestal el número con que se le señaló en 1804, que era el $114^{44}$.

Al igual que sucede en el caso del retrato antes citado-cuyo pedestal es idéntico al que éste ostenta-, el escultor ha tratado de acentuar los

44 RASF, 617/3, Inventario 1804, f. 120 , 
rasgos más peculiares del personaje: así, su nariz aguileña y la boca de labios finos y apretados, marcando además la flacidez de las mejillas característica del ministro navarro. Múzquiz había nacido en 1719 , así que al morir tenía 66 años, pero debia dar la sensación de mucha más edad, tal como aparece en la estampa que hizo Selma según el retrato de Goya ${ }^{45}$. En todo caso, el rostro del político está captado con expresión bondadosa y rasgos más agradables que los de Figueroa, de acuerdo con el buen natural que parece emanar del personaje en la citada lámina.

Como el busto del Gobernador del Consejo, debe tratarse del modelo para una obra en mármol, si bien desconocemos en este caso cuál pudo ser el destino definitivo de la escultura; quizá fue hecha para la tumba de Múzquiz, aunque nada sabemos de ella, de su situación y sus características.

5. RETRATO DE JOSÉ MOÑINO, CONDE DE FLORIDABLANCA. 1791 (Figs. 8 y 9 ).

Busto en mármol blanco y peana de jaspe anaranjado.

$47 \times 28 \times 21 \mathrm{~cm}$.

Buen estado de conservación con pequeñas muescas en la peana.

Grabado a buril al dorso: Año de 1791/ s.p. Díaz.

Museo del Prado, E-451.

Procedencia: Museo Iconográfico.

Cabeza y busto con hombros en oblicuo y corte casi semicircular; lleva peluca rizada que deja ver casi completas las orejas y viste casaca con corbatín de pliegues simples.

La ficha de inventario del Museo del Prado más antigua que conserva el expediente de esta pieza (posterior a 1936) indica su procedencia del Museo Iconográfico, sin que sepamos cómo llegó a formar parte del mismo. La mayor parte de las piezas de la también llamada Iconoteca se guardaron en el edificio del Museo de Prado y allí se exhibieron desde 1879 por un periodo de algo más de diez años; al abandonarse el proyecto, sus futuros fondos se entregaron en parte al Prado y a la Biblioteca Nacional ${ }^{46}$.

La ficha titula la pieza "Busto del arquitecto Juan de Villanueva» y transcribe la inscripción interpretando las iniciales s.p. como sculpsit (es-

45 Gassier, P., Goya, Colonia 1994, n." 1215, ilustr. 313. Data la estampa entre 1783 y 1785

46 Paz Ricardo, N. de la, La lconoteca Nacional, un museo que no pudo consolidarse (www.ucm.es/info/qma/iconoteca html; Área de Arqueologia; proyecto de recopilación sistemática de fondos documentales de museos, n." PB 97-0286). 

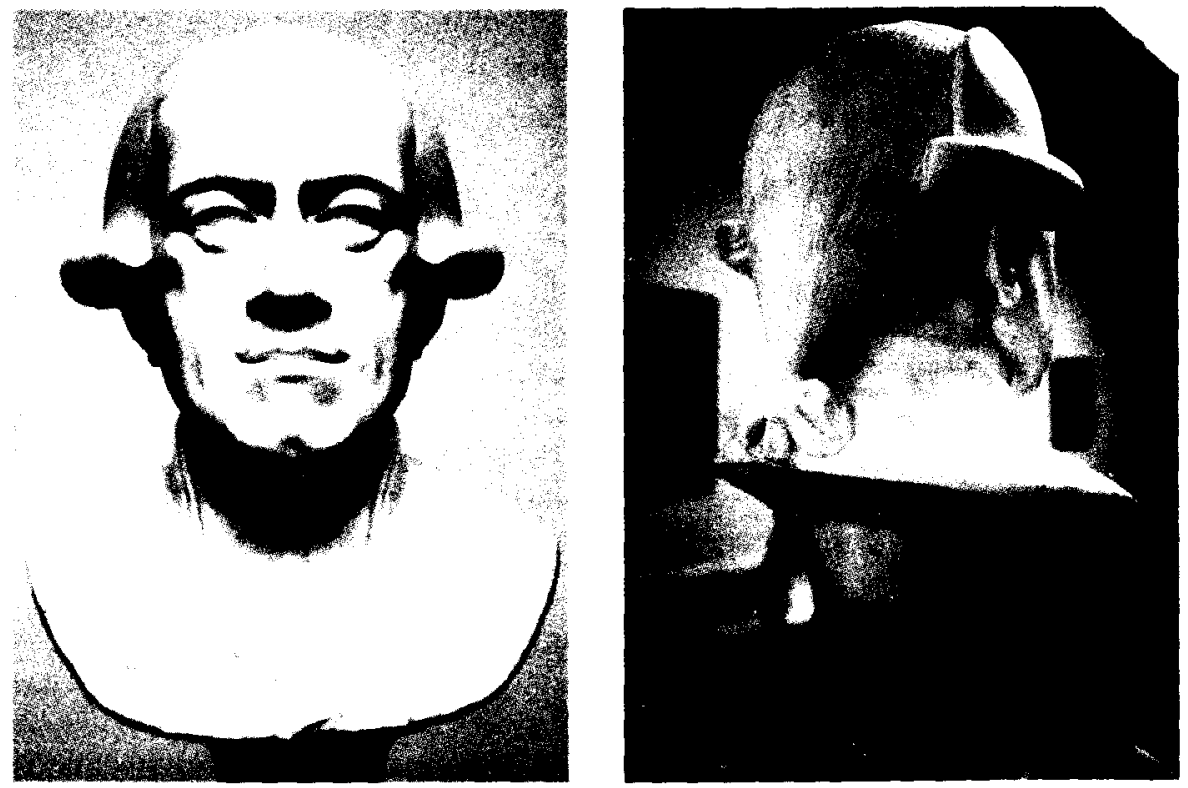

Figs. 8 y 9. José Rodriguez Díaz. Retrato de José Moñino, conde de Floridablanca. 1791 (Museo del Prado).

culpió). La obra estaba ubicada cuando se redactó esta ficha en la sala $n .^{\circ}$ 91, dedicada al legado Fernández Durán.

El Catálogo de la escultura del Museo de 1969 de Blanco y Lorente omitió la ficha de este busto, aunque lo mencionó dentro de la correspondiente a la pieza 450 , que es el retrato de Juan de Villanueva que hizo José Grajera a fines del siglo xix. Como último párrafo de esta ficha, se decía: “Otro busto del arquitecto, ejecutado en mármol, firmado en 1791 por un desconocido Díaz, se conserva también en el Museo, en la vitrina donde se exponen los planos originales de la obra». El texto se reproduce sin variaciones en la segunda edición del Catálog̣o de $1981^{47}$.

Miguel Ángel Elvira rectificó recientemente la identificación del retratado, reconociendo en él a José Moñino, conde de Floridablanca. Después de haber pasado bastantes años en la zona de dirección, la escultura ha sido colocada en la sala 88 del Museo, dentro de las nuevas salas de pined. 231

47 Blanco, A. y LoRente, M., Catálogo de escultura, Madrid 1969, 223-224; Madrid 1981, 2 . $^{a}$ 
tura española del siglo XVIII, y más recientemente aún, se le ha emparejado con el retrato de Floridablanca pintado por Goya, lo que permite observar el parecido de ambas efigies ${ }^{48}$.

Sin embargo, su autor no ha sido nunca identificado a pesar de que grabó su apellido. En nuestra opinión, no cabe duda de que se trata de José Rodríguez Díaz, no sólo por similitudes formales con los bustos que se conservan en la Academia, sino porque el escultor debía firmar así sus obras en estos años. Ponz, al dar cuenta del retrato que hizo para la tumba de Ventura Figueroa en San Martín, le denomina Joseph Díaz. Se ha de tener en cuenta que existía otro escultor formado también en la Academia, madrileño, más joven que Rodríguez Díaz, que se llamaba José Rodríguez y que por estos años ejercía ya ${ }^{49}$, por lo que quizá empezase el lucense a omitir su primer apellido al firmar sus obras, para no dar lugar a confusiones.

Este retrato adolece de mayor frialdad que los dos anteriormente comentados, lo que puede deberse a la menor ductilidad del mármol respecto del yeso y también al rostro poco expresivo del retratado. Consigue en él una perfecta imagen neoclásica en que el personaje, aunque reconocible por sus rasgos, es objeto de una cierta idealización. Parece que puede tratarse de un retrato realizado a partir de una pintura o escultura anterior, muy propio para lucir en despachos oficiales como los de la dirección de Correos, a la que se hallaba especialmente vinculado Moñino, o de algún otro ministerio. En 1791, el poder de Campomanes estaba a punto de declinar y este retrato será uno de los últimos frutos de la protección de que gozó el escultor lucense.

48 Elvira, M. A., La escultura en las salas de pintura española del sigio xvm en Guia. Goyay la pintura española del siglo xvm, Madrid 2000, 68

49 Ossorio y Bernard, M., Galería Biográfica de Artistas españoles del siglo xix, Madrid 18831884, ed. Madrid 1984, 589. El autor atribuye a José Rodriguez Díaz el Ganimedes, obra que hizo José Rodriguez para el concurso de la Academia de 1781 en la tercera clase, mientras el lucense participó con su obra Alegoria del nacimiento del infante Carlos Eusebio en la primera clase; José Rodríguez era madrileño, tenia entonces 18 años y Rodríguez Díaz 35; fue también académico de mérito según indica el Inventario de 1804, año en que estaba vivo, y posible autor de una Santa Bárbara para los Escolapios de Lavapiés (Ponz, op. cit., V, 82-83, nota 1). También le nombra como Joseph Diaz el académico González de Sepúlveda en su Diario al narrar la sesión de elección de cargos de 2 de abril de 1797 (FNMT, Diario cit., libro IV, 1797-1802, f. 18). 